\title{
Clinical impact of baseline chronic kidney disease in patients undergoing transcatheter or surgical aortic valve replacement
}

\author{
Andres M. Pineda $\mathrm{MD}^{1} \odot \mid$ J. Kevin Harrison $\mathrm{MD}^{2} \mid$ Neal S. Kleiman $\mathrm{MD}^{3}$ | \\ Michael J. Reardon MD $^{4}$ | John V. Conte MD $^{5}$ | Daniel P. O'Hair MD ${ }^{6}$ | \\ Stanley J. Chetcuti MD ${ }^{7}$ | Jian Huang MD $^{8}$ | Steven J. Yakubov MD ${ }^{9}$ (1) | \\ Jeffrey J. Popma MD ${ }^{10} \mid$ Nirat Beohar MD ${ }^{11}$
}

${ }^{1}$ Division of Cardiology, University of Florida College of Medicine-Jacksonville, Jacksonville, Florida

${ }^{2}$ Department of Medicine, Duke University Medical Center, Durham, North Carolina

${ }^{3}$ Department of Cardiology, Houston Methodist DeBakey Heart and Vascular Center, Houston, Texas

${ }^{4}$ Department of Cardiothoracic Surgery, Houston Methodist DeBakey Heart and Vascular Center, Houston, Texas

${ }^{5}$ Division of Cardiac Surgery, Johns Hopkins University, Baltimore, Maryland

${ }^{6}$ Department of Cardiothoracic Surgery, Aurora Healthcare, Milwaukee, Wisconsin

${ }^{7}$ Department of Internal Medicine, Division of Cardiology, University of Michigan, Ann Arbor, Michigan

${ }^{8}$ Coronary and Structural Heart Clinical Department, Mounds View, Minnesota

${ }^{9}$ Department of Cardiology, Riverside Methodist Hospital, Columbus, Ohio

${ }^{10}$ Department of Internal Medicine, Cardiovascular Division, Beth Israel Deaconess Medical Center, Boston, Massachusetts

${ }^{11}$ Division of Cardiology at Mount Sinai Medical Center, Columbia University, Miami Beach, Florida

Correspondence

Nirat Beohar, MD, FACC, FSCAI, Professor, Columbia University Medical Center, Director, Cardiac Catheterization Laboratory, Suite 2070, de Hirsch Meyer Tower, Miami Beach, FL 33140.

Email: nirat.beohar@msmc.com

Funding information

Medtronic (Minneapolis, MN)

\begin{abstract}
Objectives: To assess the treatment effect of TAVR versus SAVR on clinical outcomes to 3 years in patients stratified by chronic kidney disease (CKD) by retrospectively studying patients randomized to TAVR or SAVR.

Background: The impact of CKD on mid-term outcomes of patients undergoing TAVR versus SAVR is unclear.

Methods: Patients randomized to TAVR or SAVR in the CoreValve US Pivotal High Risk Trial were retrospectively stratified by eGFR: none/mild or moderate/severe CKD. To evaluate the impact of baseline CKD in TAVR patients only, all patients undergoing an attempted TAVR implant in the US Pivotal Trial and CAS were stratified by baseline eGFR into none/mild, moderate, and severe CKD. The primary endpoint was major adverse cardiovascular and renal events (MACRE), a composite of all-cause mortality, myocardial infarction, stroke/TIA, and new requirement of dialysis.

Results: Moderate/severe CKD was present in $62.7 \%$ and $60.7 \%$ of high-risk patients randomized to TAVR or SAVR, respectively. Baseline characteristics were similar between TAVR and SAVR patients in both CKD subgroups, except for higher rates of diabetes and higher serum creatinine in SAVR patients. Among high-risk patients with moderate/severe CKD, TAVR provided a lower 3-year MACRE rate compared with SAVR: $42.1 \%$ vs. $51.0, P=.04$. Of 3,733 extremeand high-risk TAVR patients, $39.9 \%$ had none/mild, 53.8\% moderate, and $6.4 \%$ severe CKD. Worsening baseline CKD was associated with increased 3-year MACRE rates [none/mild 51.5\%, moderate $54.5 \%$, severe $63.1 \%, P=.001]$.

Conclusions: TAVR results in lower 3-year MACRE versus SAVR in high-risk patients with moderate/severe CKD. In patients undergoing TAVR, worsening CKD increases mid-term mortality and MACRE. Randomized trials of TAVR vs. SAVR in patients with moderate-severe CKD would help elucidate the best treatment for these complex patients.

Trial Registration: CoreValve US Pivotal Trial: NCT01240902.

CoreValve Continued Access Study: NCT01531374.
\end{abstract}

\section{KEYWORDS}

aortic stenosis, renal disease, SAVR, TAVR 


\section{1 | INTRODUCTION}

The presence of advanced chronic kidney disease (CKD) significantly worsens the short- and mid-term clinical outcomes of patients undergoing surgical aortic valve replacement (SAVR) for severe aortic stenosis (AS), with operative mortality as high as $21 \%^{1}$. Transcatheter aortic valve replacement (TAVR) has emerged as a less invasive alternative for patients with severe $A S^{2,3}$.

The feasibility of TAVR or SAVR in patients with CKD has been shown in observational studies ${ }^{4-10}$. Worsening CKD has been shown to be associated with higher incidence of major bleeding and vascular complications, as well as increased all-cause mortality rates ${ }^{11-13}$. The impact of CKD on the mid-term outcomes of patients undergoing TAVR versus SAVR is not known.

The primary aim of this study was to assess the treatment effect of TAVR versus SAVR on the clinical outcomes to 3 years of patients with varying degrees of CKD randomized in the CoreValve US Pivotal High Risk Trial. The secondary aim was to assess the impact of CKD on clinical outcomes to 3 years in all extreme-risk and high-risk patients undergoing TAVR as part of the CoreValve US Pivotal Trial and Continued Access Study (CAS).

\section{2 | METHODS}

\section{1 | Study design}

The CoreValve US Pivotal Trial was a prospective, controlled, multicenter clinical trial conducted in patients with severe symptomatic AS and extreme- or high-surgical risk at 45 sites in the United States. Details of the study design and methodology have been previously published $^{14,15}$. At each site, the institutional review board approved the study protocol, and all patients provided written informed consent. The present analysis comprises extreme- and high-risk patients from the CoreValve US Pivotal Extreme Risk Trial, Pivotal High Risk Trial, and CAS as further described below.

\section{2 | Patient selection}

Trial inclusion/exclusion criteria have been previously published ${ }^{14,15}$. Patients randomized in the US Pivotal High Risk Trial to TAVR or SAVR who received an attempted implant were stratified into none/ mild or moderate/severe CKD according to their baseline renal function and their outcomes were evaluated. The Modification of Diet in Renal Disease equation was used to calculate the estimated glomerular filtration (eGFR) from the patient's age, sex, race and baseline serum creatinine. Patients without available baseline renal function data were excluded from this analysis. Patients with end-stage renal disease requiring chronic dialysis or those with an eGFR of $\leq 20 \mathrm{~mL} / \mathrm{min} / 1.73 \mathrm{~m}^{2}$ were excluded from the CoreValve US Pivotal Trial and $\mathrm{CAS}^{14,15}$. Due to the small number of patients with severe CKD precluding meaningful analysis if evaluated separately, they were combined with those with moderate CKD and only two groups, none/mild (eGFR $>60 \mathrm{~mL} / \mathrm{min} / 1.73 \mathrm{~m}^{2}$ ) or moderate/severe (eGFR $\left.\leq 60 \mathrm{~mL} / \mathrm{min} / 1.73 \mathrm{~m}^{2}\right)$, were evaluated.
To evaluate the impact of baseline CKD in TAVR patients only, all patients undergoing an attempted TAVR implant in the US Pivotal Trial and CAS were stratified by baseline eGFR into none/mild (eGFR $>60 \mathrm{~mL} / \mathrm{min} / 1.73 \mathrm{~m}^{2}$ ), moderate (eGFR $30-60 \mathrm{~mL} / \mathrm{min} / 1.73$ $\mathrm{m}^{2}$ ), and severe (eGFR<30 mL/min/1.73 $\left.\mathrm{m}^{2}\right) \mathrm{CKD}$ and their clinical outcomes assessed.

\section{3 | Study endpoints}

The primary endpoint is the composite of major adverse cardiovascular and renal events (MACRE) at 3 years, which included all-cause mortality, any stroke/TIA, myocardial infarction, and new requirement of dialysis. Individual components of MACRE and other clinical endpoints are also reported. Definitions of clinical endpoints are based on the Valve Academic Research Consortium ${ }^{14-16}$.

\section{4 | Statistical analysis}

Categorical variables were compared using the Fisher exact test or the chi-square test. Continuous variables are presented as mean \pm standard deviation, with a $t$-test used for comparisons between two groups and ANOVA used for comparisons across 3 groups. The KaplanMeier estimates were used to construct survival and MACRE curves based on all available follow-up data for the time-to-event analysis. Differences in event rates were evaluated using the log-rank test. All testing used a 2 -sided $\alpha$ level of 0.05 . For the predictors of mortality analysis, variables were selected based on clinical relevance. Cox proportional hazards survival models were used for 1-year mortality univariable and multivariable modeling analysis. Variables for inclusion in the Cox model for 1-year mortality were selected based on clinical relevance (Supporting Information Table S1). Multivariable predictors were identified from univariable predictors with $P \leq .05$. A stepwise procedure was performed to determine the final model. The significance level thresholds for entry and exit of independent variables were set at 0.10. Statistical analyses were performed with the use of SAS software, version 9.2 or above (SAS Institute, Cary, NC).

\section{3 | RESULTS}

\section{1 | High-risk patient characteristics}

Patients randomized as part of the US Pivotal High Risk Trial were stratified according to their CKD (none/mild vs. moderate/severe) to compare the treatment effect of TAVR versus SAVR on clinical outcomes (Figure 1). Among 871 patients enrolled, 797 underwent randomization, with 391 patients among the attempted TAVR group and 359 patients in the attempted SAVR group. Moderate/severe CKD was present in $60.7 \%$ of high-risk patients randomized to SAVR and in $62.7 \%$ of patients randomized to TAVR. Baseline characteristics were similar between TAVR and SAVR patients in both CKD subgroups, except for higher rates of diabetes and higher serum creatinine in SAVR patients and a higher frequency of hostile mediastinum among TAVR patients in the none/mild CKD group (Table 1). 


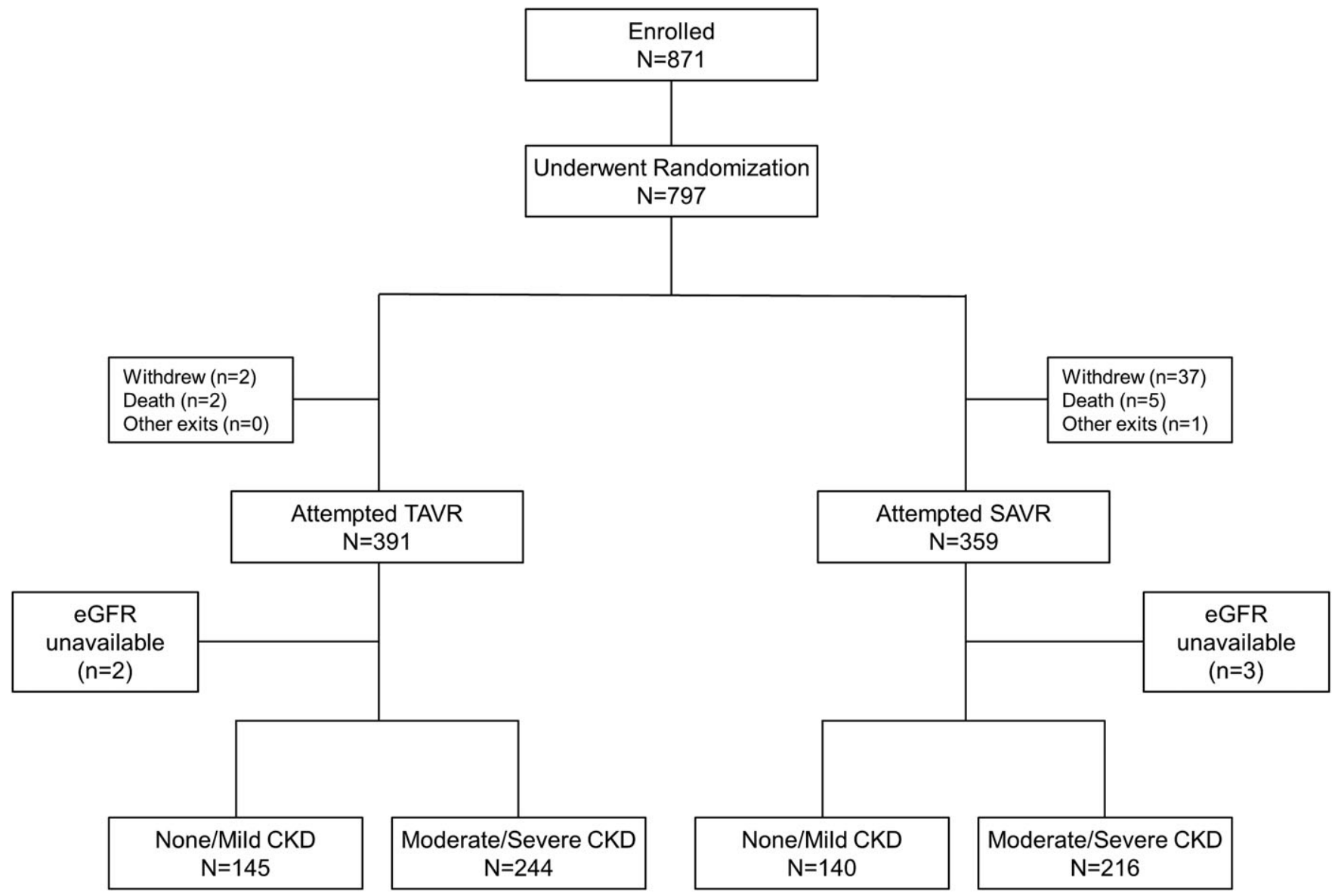

FIGURE 1 Patient flow diagram for the high-risk analysis. The "attempted TAVR" and "attempted SAVR" cohorts include all patients randomized to TAVR or SAVR who received an attempted implant (also includes intraprocedural deaths or other patients who may not have received an implant). Abbreviations: CKD: chronic kidney disease; eGFR: estimated Glomerular Filtration Rate

\section{2 | Clinical outcomes after TAVR or SAVR}

At 3-year follow up, the MACRE rate was lower in TAVR patients compared with SAVR patients in the moderate/severe CKD group (42.1\% vs. $51.0 \%, P=.04$ ) but not in the none/mild CKD group. There were no differences in all-cause mortality, MI or all stroke/TIA or need for new dialysis among groups. AKI was less frequent among TAVR as compared with SAVR patients in both the none/mild $(0.7 \%$ vs. $10.7 \%$, $P<.001)$ and moderate/severe (9.6\% vs. $18.2 \%, P=.001) C K D$ groups. Life-threatening or disabling bleeding was lower among TAVR compared with SAVR patients in both none/mild (15.8\% vs. $33.7 \%$, $P<.001)$ and moderate/severe $(20.7 \%$ vs. $46.4 \%, P<.001)$ CKD groups. Major vascular complications and need for new permanent pacemaker were more frequent among TAVR compared with SAVR patients in both CKD groups (Table 2, Figure 2).

\section{3 | TAVR patient characteristics}

To look at TAVR patients in isolation, 3,733 extreme- and high-risk patients who underwent TAVR in the CoreValve US Pivotal Trial and CAS (Supporting Information Figure S1) were stratified according to their baseline eGFR: none/mild ( $n=1,488,39.9 \%$ ), moderate ( $n=2,007,53.8 \%$ ), or severe $(n=238,6.4 \%$ ) CKD. Baseline clinical and echocardiographic as well as procedural characteristics are presented in Table 3. Patients in the none/mild CKD group were younger, and male sex and hostile mediastinum were more frequent. Patients in the severe CKD group had a higher mean STS-PROM and logistic EuroSCORE, and a higher incidence of comorbidities than patients with none/mild or moderate CKD (Table 3).

\section{4 | Clinical outcomes: TAVR patients}

Worsening CKD was associated with a higher rate of MACRE at 3 years (Table 4). The difference in MACRE was driven predominantly by an increase in new requirement for dialysis and the all-cause mortality rate in patients with severe CKD. The 3-year MACRE (none/mild 51.5\%, moderate 54.5\%, severe CKD 63.1\%, log-rank $P=.001$ ) and all-cause mortality (none/mild $44.1 \%$, moderate $46.1 \%$, severe CKD $56.0 \%, \log$-rank $P=.01$ ) rates were significantly higher for the severe CKD group (Figure 3). Three-year rates of new requirement of dialysis, cardiovascular mortality, major bleeding, and acute kidney injury were significantly higher in patients with severe CKD (Table 4).

\section{5 | Predictors of 1-year all-cause mortality for TAVR patients with moderate/severe CKD}

A Cox regression model was built to identify covariates associated with 1-year all-cause mortality in patients with moderate/severe CKD. Albumin $<3.3 \mathrm{~g} / \mathrm{dL}$, male sex, BMI $<21 \mathrm{~kg} / \mathrm{m}^{2}$, falls in the past 6 months, home oxygen, assisted living residents, baseline moderate/ 
TABLE 1 Baseline clinical characteristics in high-risk patients, TAVR vs. SAVR

\begin{tabular}{|c|c|c|c|c|c|c|}
\hline \multirow[b]{3}{*}{ Demographics } & \multicolumn{3}{|c|}{ None/mild CKD } & \multicolumn{3}{|c|}{ Moderate/severe CKD } \\
\hline & \multicolumn{3}{|l|}{$\mathrm{N}=285$} & \multicolumn{3}{|l|}{$\mathrm{N}=460$} \\
\hline & $\begin{array}{l}\text { TAVR } \\
(\mathrm{N}=145)\end{array}$ & $\begin{array}{l}\text { SAVR } \\
(\mathrm{N}=140)\end{array}$ & $P$-value & $\begin{array}{l}\text { TAVR } \\
(\mathrm{N}=244)\end{array}$ & $\begin{array}{l}\text { SAVR } \\
(\mathrm{N}=216)\end{array}$ & $P$-value \\
\hline Age (years) & $82.5 \pm 7.9$ & $82.7 \pm 6.9$ & 0.75 & $83.5 \pm 6.6$ & $83.7 \pm 5.9$ & 0.81 \\
\hline Body surface area $\left(\mathrm{m}^{2}\right)$ & $1.8 \pm 0.2$ & $1.9 \pm 0.3$ & 0.50 & $1.9 \pm 0.2$ & $1.9 \pm 0.2$ & 0.88 \\
\hline Men & 78 (53.8\%) & $83(59.3 \%)$ & 0.35 & 129 (52.9\%) & $102(47.2 \%)$ & 0.23 \\
\hline STS score (risk of mortality, \%) & $6.2 \pm 2.6$ & $6.5 \pm 2.9$ & 0.42 & $7.9 \pm 3.1$ & $8.2 \pm 3.4$ & 0.36 \\
\hline Diabetes mellitus & 39 (26.9\%) & $64(45.7 \%)$ & 0.001 & 96 (39.3\%) & 97 (44.9\%) & 0.23 \\
\hline Serum creatinine (mg/dl) & $0.8 \pm 0.2$ & $0.9 \pm 0.2$ & 0.04 & $1.4 \pm 0.4$ & $1.4 \pm 0.4$ & 0.96 \\
\hline Hypertension & $136(93.8 \%)$ & 131 (93.6\%) & 0.94 & 234 (95.9\%) & 211 (97.7\%) & 0.28 \\
\hline Peripheral vascular disease & $60(41.4 \%)$ & $61(43.6 \%)$ & 0.71 & 98 (40.7\%) & 87 (40.7\%) & $>0.99$ \\
\hline Coronary artery bypass surgery & 45 (31.0\%) & $40(28.6 \%)$ & 0.65 & 69 (28.3\%) & 71 (32.9\%) & 0.29 \\
\hline Percutaneous coronary intervention & 47 (32.4\%) & 48 (34.3\%) & 0.74 & 85 (34.8\%) & $87(40.3 \%)$ & 0.23 \\
\hline Balloon valvuloplasty & $6(4.1 \%)$ & $11(7.9 \%)$ & 0.19 & $17(7.0 \%)$ & $11(5.1 \%)$ & 0.40 \\
\hline Previous myocardial infarction & 35 (24.1\%) & $36(25.7 \%)$ & 0.76 & $63(25.8 \%)$ & $54(25.0 \%)$ & 0.84 \\
\hline Congestive heart failure & 141 (97.2\%) & $133(95.0 \%)$ & 0.37 & 231 (94.7\%) & 211 (97.7\%) & 0.10 \\
\hline Atrial fibrillation/atrial flutter & 51 (35.2\%) & $58(41.4 \%)$ & 0.28 & 109 (44.9\%) & $106(49.1 \%)$ & 0.37 \\
\hline Chest wall deformity & $6(4.1 \%)$ & $1(0.7 \%)$ & 0.12 & $3(1.2 \%)$ & $0(0.0 \%)$ & 0.25 \\
\hline Hostile mediastinum & $8(5.5 \%)$ & $1(0.7 \%)$ & 0.04 & $7(2.9 \%)$ & $4(1.9 \%)$ & 0.55 \\
\hline
\end{tabular}

Abbreviation: COPD, chronic obstructive pulmonary disease.

Continuous data are presented as mean \pm standard deviation. Categorical data are presented as no. (\%) reflecting missing data.

severe baseline mitral regurgitation, peripheral vascular disease, prior stroke/ TIA, moderate/severe baseline tricuspid regurgitation, STS risk score $>7 \%$, and estimated volume of iodinated contrast media used (per $10 \mathrm{cc}$ ) were found to be predictors of 1-year all-cause mortality. Patients undergoing ilio-femoral access were less likely to die after 1 year (Figure 4).

TABLE 2 Three-year outcomes in high-risk patients, TAVR vs. SAVR

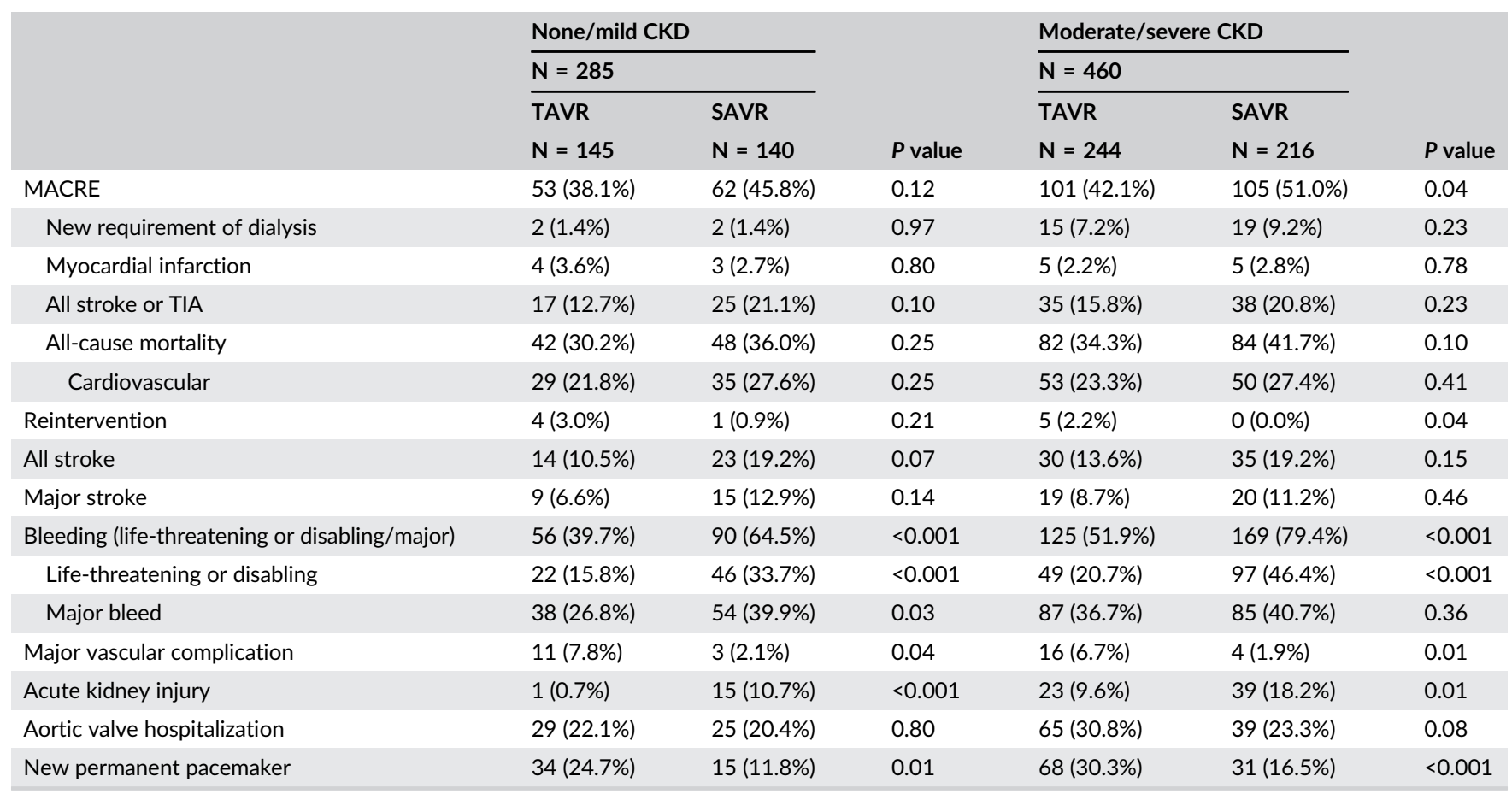

Abbreviations: CKD, chronic kidney disease; MACRE, major adverse cardiovascular and renal events; TIA, transient ischemic attack.

Values are Kaplan-Meier estimates and are reported as no. of patients with the event (\%). P-value is calculated based on Log-rank test. 
TABLE 3 Baseline demographics and procedural characteristics, all TAVR patients

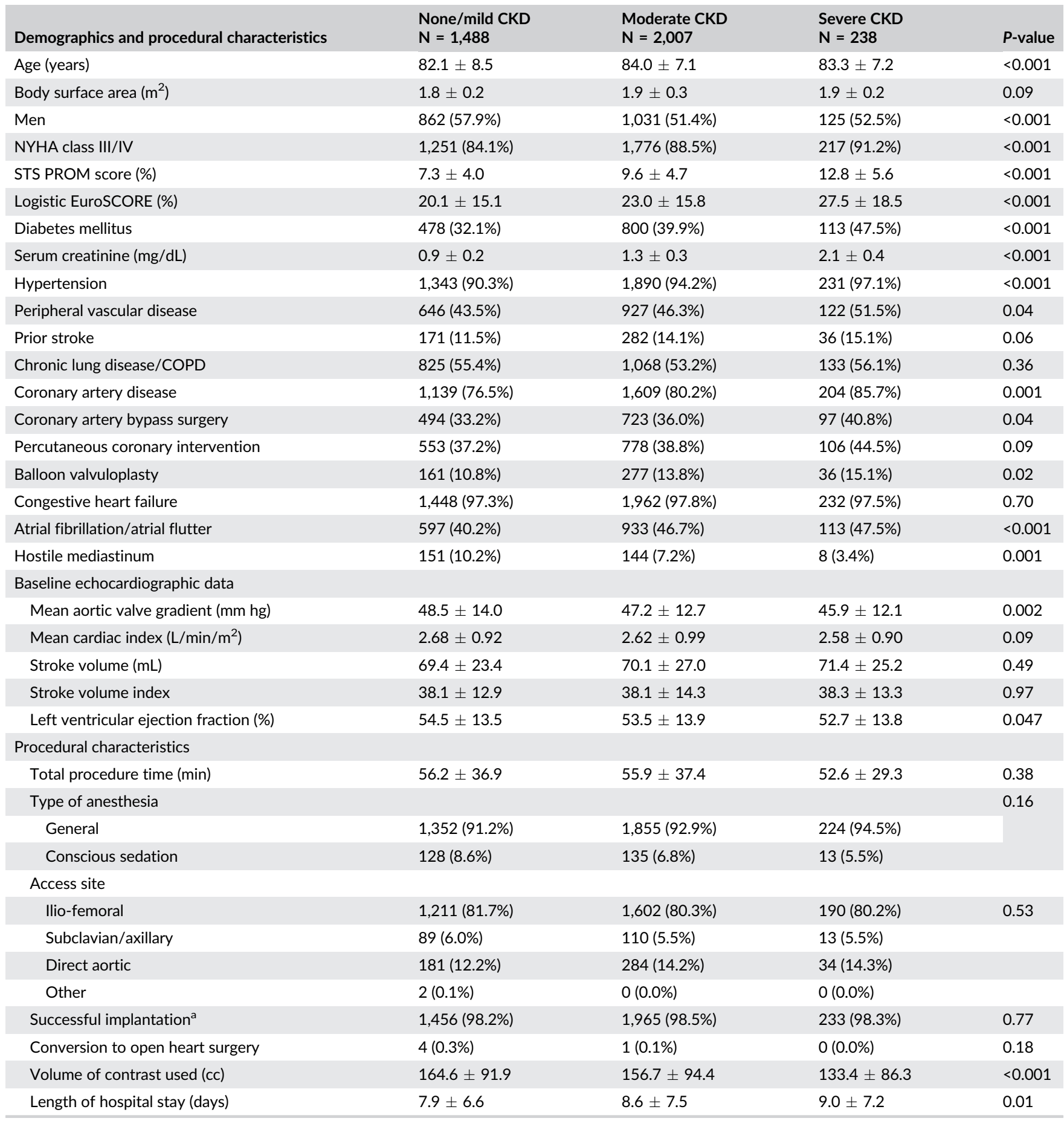

Abbreviations: CKD, chronic kidney disease; COPD, chronic obstructive pulmonary disease.

Continuous data are presented as mean \pm standard deviation. Categorical data are presented as no. (\%), reflecting missing data.

${ }^{a}$ Successful vascular access, delivery and deployment of the device.

\section{DISCUSSION}

The principal findings of this analysis are: (1) moderate to severe CKD was frequent, being present in over $60 \%$ of high or extreme-risk TAVR and high-risk SAVR patients in the present study; (2) in high-risk patients with moderate/severe CKD, TAVR provided better clinical outcomes when compared with SAVR, with a significantly lower 3-year rate of MACRE; (3) in high- risk patients who underwent TAVR, severe CKD was associated with an increased incidence of the mid-term adverse clinical outcomes, including all-cause mortality and MACRE; (4) several baseline clinical and some procedural features were found to be associated with the 1-year all-cause mortality among high- or extreme-risk patients with moderate/severe CKD undergoing TAVR.

Consistent with others, we found a high frequency of moderate/severe CKD among the high- to extreme-risk patients undergoing TAVR and high-risk patients randomized to SAVR ${ }^{11,17}$, which is likely to be multifactorial ${ }^{11,12,18-20}$. While previous 

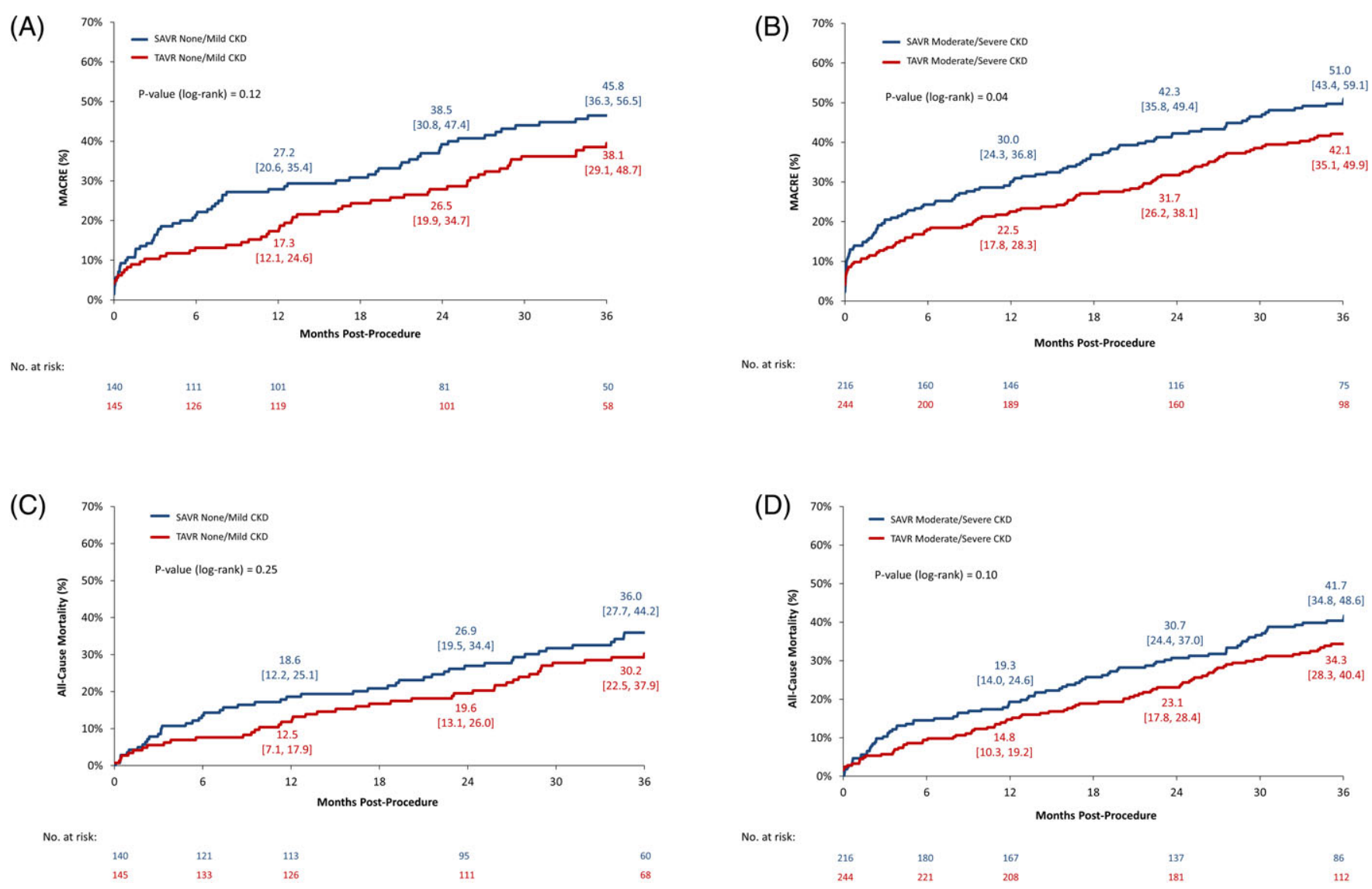

FIGURE 2 MACRE and all-cause mortality rates, TAVR vs. SAVR in high-risk patients. MACRE composite outcome in high-risk TAVR (red lines) vs. SAVR (blue lines) patients with none/mild CKD (A) and moderate/severe CKD (B), and all-cause mortality in TAVR (red lines) vs. SAVR (blue lines) for patients with none/mild CKD (C) and moderate/severe CKD (D). Values are KM \% (95\% CI)

studies $^{11,12,18}$ provided short-term outcomes, the UK TAVI registry ${ }^{18}$ and the present study provide mid-term (3-year) outcomes. Additionally, unlike previous studies ${ }^{12,18,20}$, our analysis specifically evaluated the impact of CKD on the outcomes of high- and extreme-risk patients undergoing TAVR similar to one prior study from the PARTNER 1 trial $^{11}$.

TABLE 4 Three-year outcomes, all TAVR patients

\begin{tabular}{|c|c|c|c|c|}
\hline & $\begin{array}{l}\text { None/mild CKD } \\
\mathrm{N}=1,488\end{array}$ & $\begin{array}{l}\text { Moderate CKD } \\
N=2,007\end{array}$ & $\begin{array}{l}\text { Severe CKD } \\
N=238\end{array}$ & $P$ value \\
\hline MACRE & $624(51.5 \%)$ & $914(54.5 \%)$ & $125(63.1 \%)$ & 0.001 \\
\hline New requirement of dialysis & $24(2.1 \%)$ & $90(5.9 \%)$ & $27(15.8 \%)$ & $<0.001$ \\
\hline Myocardial infarction & 48 (4.7\%) & $50(3.7 \%)$ & $10(5.1 \%)$ & 0.17 \\
\hline All stroke or TIA & $173(14.8 \%)$ & $261(17.8 \%)$ & $29(17.1 \%)$ & 0.44 \\
\hline All-cause mortality & $514(44.1 \%)$ & 747 (46.1\%) & 103 (56.0\%) & 0.01 \\
\hline Reintervention & 20 (1.5\%) & $22(1.3 \%)$ & $3(1.3 \%)$ & 0.81 \\
\hline All stroke & $140(11.8 \%)$ & $215(14.7 \%)$ & $25(15.0 \%)$ & 0.39 \\
\hline Major stroke & $82(7.4 \%)$ & $128(8.9 \%)$ & 15 (8.8\%) & 0.51 \\
\hline Bleeding (life-threatening or disabling/major) & $590(42.4 \%)$ & $901(47.5 \%)$ & 122 (57.0\%) & $<0.001$ \\
\hline Life-threatening or disabling & $246(18.5 \%)$ & $357(20.3 \%)$ & $46(22.2 \%)$ & 0.41 \\
\hline Aortic valve hospitalization & $268(24.8 \%)$ & 491 (33.4\%) & 71 (44.9\%) & $<0.001$ \\
\hline New permanent pacemaker & $362(27.6 \%)$ & $555(31.2 \%)$ & $64(34.2 \%)$ & 0.08 \\
\hline
\end{tabular}

Abbreviations: CKD, chronic kidney disease; MACRE, major adverse cardiovascular and renal events; TIA, transient ischemic attack.

Values are Kaplan-Meier estimates and are reported as no. of patients with the event (\%). P-value is calculated based on Log-rank test. 

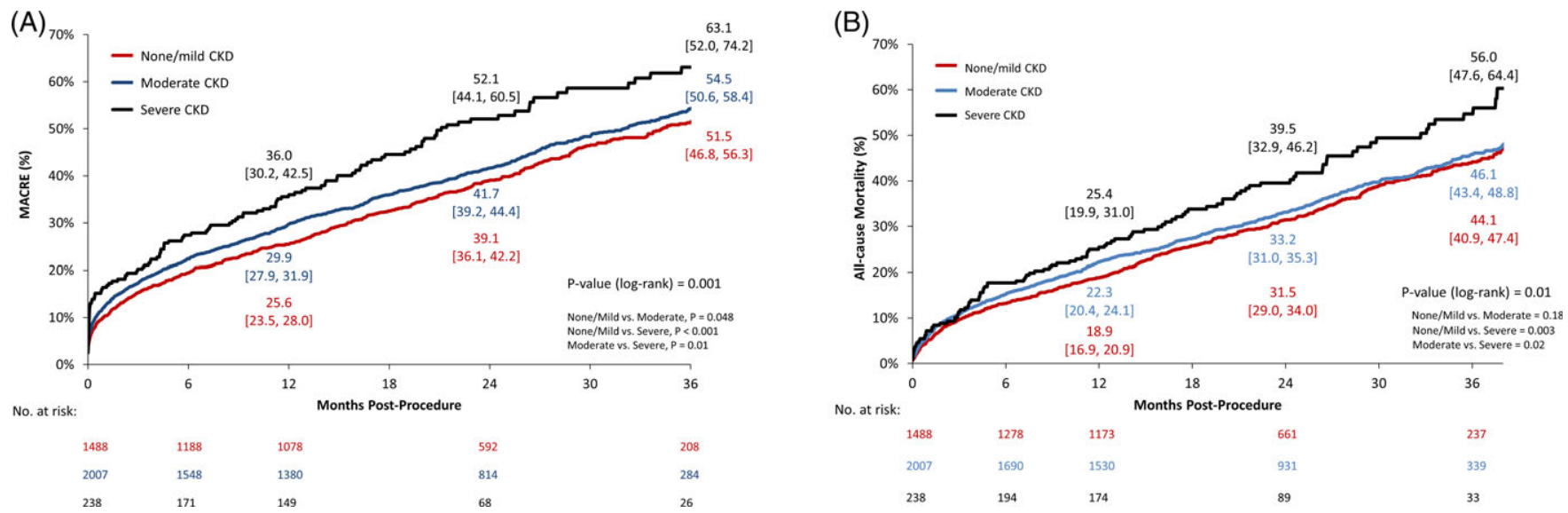

FIGURE 3 MACRE composite endpoint (A) and all-cause mortality (B) for all TAVR patients up to 3 years post-procedure. Patients were stratified by none/mild (red lines), moderate (blue lines), or severe (black lines) baseline CKD. Values are KM \% $(95 \% \mathrm{Cl})$

The composite endpoint of MACRE is one of the unique features of the present analysis. The MACRE rate was significantly increased with worsening CKD up to 3 years after TAVR and driven by an increase in all-cause mortality and new requirement for dialysis. We also found increased new requirement for dialysis according to CKD. The risk of $\mathrm{AKI}$ increased as CKD worsened and likely contributed to the new requirement of dialysis rate. These results are consistent with previous studies which reported only short-term data ${ }^{11}$.

As TAVR has emerged as an effective therapy for symptomatic severe AS, the question of offering TAVR rather than SAVR to patients with CKD has become increasingly important. We found a significantly lower 3-year rate of MACRE with TAVR compared with SAVR in patients with moderate/severe CKD $(P=.04)$. The rates of bleeding (major/life-threatening or disabling) and AKI were consistently higher for SAVR patients. The higher rates of bleeding among SAVR patients may have also resulted in a higher AKI rate. Previous results from the CoreValve High Risk Pivotal Trial demonstrated increased mortality in SAVR patients with AKI, but not TAVR patients with $A K I^{13}$. The increase in bleeding rates in SAVR cases may be related to the platelet dysfunction associated with renal disease, with a further negative impact on platelet function due to cardiopulmonary bypass. These findings suggest worse outcomes with SAVR rather than TAVR in high-risk patients with moderate to severe CKD and are consistent with a retrospective analysis not confined to high- or extreme-risk patients by Nguyen et al. ${ }^{10}$.

In patients with moderate/severe CKD, we found baseline clinical features associated with increased disease burden and frailty to be predictors of 1-year all-cause mortality, consistent with previous studies $^{11},{ }^{21}, 17$. Also consistent with others ${ }^{22,23}$, we found multi-valvular involvement including tricuspid regurgitation to be associated with 1-year all-cause mortality, thus, the presence of such multi-valvular pathology should be carefully considered in counseling patients.

We found procedural characteristics to be associated with 1-year all-cause mortality, following TAVR. Consistent with others, we found the volume of iodinated contrast used, especially in patients with baseline renal dysfunction, to be associated with increased risk of

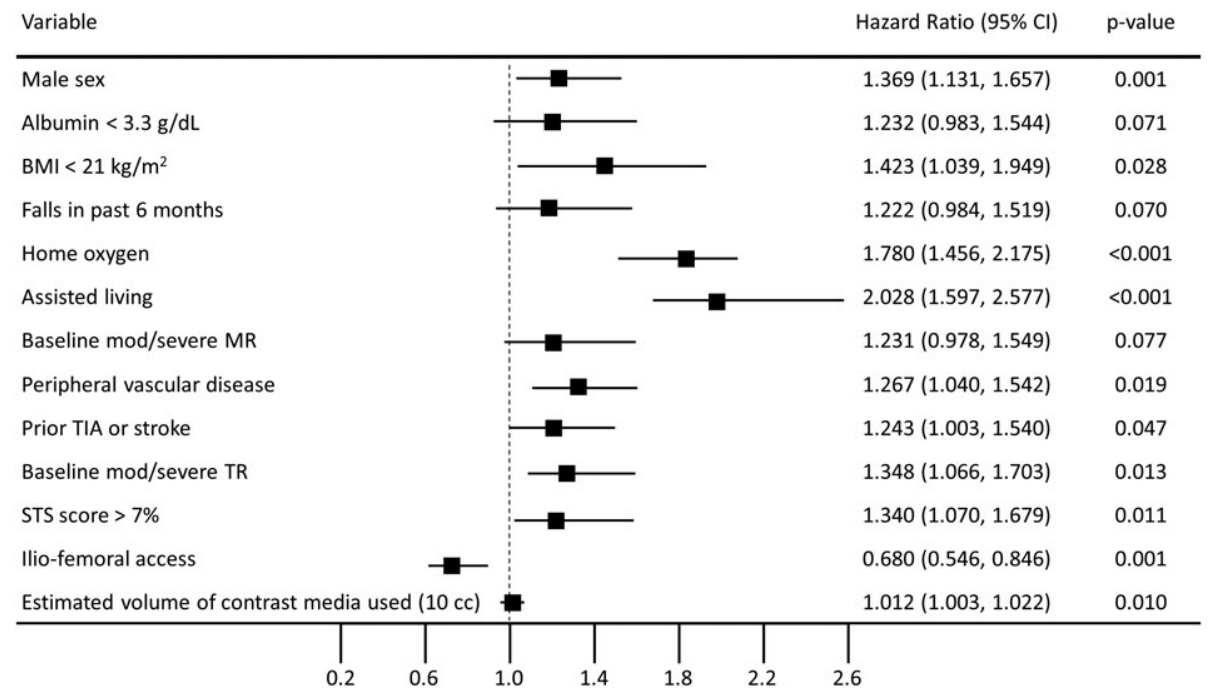

FIGURE 4 Multivariable predictors of 1-year mortality in all TAVR patients with moderate/severe CKD all TAVR patients with eGFR $\leq 60 \mathrm{~mL} /$ $\mathrm{min} / 1.73 \mathrm{~m}^{2}$ were included. Variables were selected from univariable predictors with $P$-value $\leq 0.05$. Stepwise method with thresholds for entry and exit $=0.10$ 
acute kidney injury and elevated 1-year mortality highlighting the importance of minimizing contrast use ${ }^{24-26}$. Consistent with the literature, we found a higher rate of vascular complications and the need for permanent pacemaker placement with TAVR. However, both of these complications will likely continue to decline and may not pose a disadvantage with TAVR rather than a SAVR approach ${ }^{27,28}$. Also consistent with previous literature ${ }^{11}$, we found ilio-femoral access to be protective, likely due to the less invasive nature of this approach, and the fact that those undergoing alternative access TAVR often have a higher burden of disease.

There are several limitations to consider. First, the present study was a post-hoc analysis of patients included in the CoreValve US Pivotal Extreme Risk and High Risk Trial and CAS. The number of patients in the severe CKD group was small. The new requirement of dialysis was not a prespecified data collection endpoint, and as such was manually collected and reviewed, and not adjudicated by the Clinical Events Committee. Due to a small sample size in the high-risk cohort with severe CKD, we were only able to perform a 2-group (none/mild and moderate/severe) comparison between TAVR and SAVR, which limits the applicability of our results in patients with severe CKD. Patients with end stage renal disease requiring chronic dialysis or those with an eGFR of $\leq 20 \mathrm{~mL} / \mathrm{min} / 1.73 \mathrm{~m}^{2}$ were excluded from the CoreValve US Pivotal Trial and the CAS; therefore, we were unable to provide outcomes with TAVR or SAVR for this sub-group in the present analysis.

\section{5 | CONCLUSION}

In conclusion, TAVR was found to provide a lower 3-year rate of MACRE when compared with SAVR in high-risk patients with moderate/severe CKD suggesting TAVR is the preferred strategy for the treatment of these complex patients. Additionally, in patients undergoing TAVR, worsening CKD increases short- and mid-term all-cause mortality and the incidence of MACRE. Clinical features of increased disease burden, frailty, and procedural characteristics were found to be predictors of 1-year all-cause mortality in patients with moderate/ severe CKD, emphasizing the need for a careful risk stratification and choice of procedural approach in such patients.

\section{ACKNOWLEDGMENT}

Jessica Dries-Devlin, PhD, CMPP, an employee of Medtronic, created all tables and figures, and ensured technical accuracy of the manuscript. The study was designed and funded by Medtronic (Minneapolis, MN).

\section{CONFLICT OF INTEREST}

Dr. Pineda and Dr. Beohar have no disclosures. Dr. Harrison has received institutional grants from Medtronic, Boston Scientific, Direct Flow Medical, St. Jude Medical, and Edwards Lifesciences; and serves on a Medical Advisory Board for Direct Flow Medical and on the Data Safety Monitoring Board for CardiAQ. Dr. Kleiman and Dr. Reardon have received fees from Medtronic for providing educational services.
Dr. Conte serves on a surgical advisory board for Medtronic and Sorin. Dr. O'Hair reports receiving grant support from Medtronic and Edwards Lifesciences. Dr. Chetcuti has received grant support from Edwards Lifesciences, Boston Scientific, and Medtronic, and has received proctoring fees from Medtronic. Dr. Huang is an employee and shareholder of Medtronic. Dr. Yakubov has received grant support and served on advisory boards for Medtronic and Boston Scientific. Dr. Popma has received grants from Medtronic, Boston Scientific, and Direct Flow Medical.

\section{ORCID}

Andres M. Pineda (D) https://orcid.org/0000-0002-9877-114X Steven J. Yakubov (D) https://orcid.org/0000-0002-0952-7509 Nirat Beohar (D) https://orcid.org/0000-0001-8413-9664

\section{REFERENCES}

1. Herzog CA, Ma JZ, Collins AJ. Long-term survival of dialysis patients in the United States with prosthetic heart valves: Should ACC/AHA practice guidelines on valve selection be modified? Circulation 2002; 105:1336-1341.

2. Mack MJ, Leon MB, Smith CR, et al. 5-year outcomes of transcatheter aortic valve replacement or surgical aortic valve replacement for high surgical risk patients with aortic stenosis (PARTNER 1): A randomised controlled trial. Lancet 2015;385:2477-2484.

3. Deeb GM, Reardon MJ, Chetcuti S, et al. 3-year outcomes in high-risk patients who underwent surgical or transcatheter aortic valve replacement. J Am Coll Cardiol 2016;67:2565-2574.

4. O'Hair DP, Bajwa TK, Chetcuti SJ, et al. One-year outcomes of transcatheter aortic valve replacement in patients with end-stage renal disease. Ann Thorac Surg 2017;103:1392-1398.

5. Allende R, Webb JG, Munoz-Garcia AJ, et al. Advanced chronic kidney disease in patients undergoing transcatheter aortic valve implantation: Insights on clinical outcomes and prognostic markers from a large cohort of patients. Eur Heart J 2014;35:2685-2696.

6. Conrotto F, Salizzoni S, Andreis A, et al. Transcatheter aortic valve implantation in patients with advanced chronic kidney disease. Am J Cardiol 2017;119:1438-1442.

7. D'Errigo P, Moretti C, D'Ascenzo F, et al. Transcatheter aortic valve implantation versus surgical aortic valve replacement for severe aortic stenosis in patients with chronic kidney disease stages $3 \mathrm{~b}$ to 5 . Ann Thorac Surg 2016;102:540-547.

8. Alqahtani F, Aljohani S, Boobes $\mathrm{K}$, et al. Outcomes of transcatheter and surgical aortic valve replacement in patients on maintenance dialysis. Am J Med 2017;130:1464.e1-1464.e11.

9. Kobrin DM, McCarthy FH, Herrmann HC, et al. Transcatheter and surgical aortic valve replacement in dialysis patients: A propensity-matched comparison. Ann Thorac Surg 2015;100: 1230-1236. discussion 1236-1237.

10. Nguyen TC, Babaliaros VC, Razavi SA, et al. Impact of varying degrees of renal dysfunction on transcatheter and surgical aortic valve replacement. J Thorac Cardiovasc Surg 2013;146:1399-1406. discussion 13406-13407.

11. Thourani $\mathrm{VH}$, Forcillo $\mathrm{J}$, Beohar $\mathrm{N}$, et al. Impact of preoperative chronic kidney disease in 2,531 high-risk and inoperable patients undergoing transcatheter aortic valve replacement in the PARTNER trial. Ann Thorac Surg 2016;102:1172-1180.

12. Codner P, Levi A, Gargiulo G, et al. Impact of renal dysfunction on results of transcatheter aortic valve replacement outcomes in a large multicenter cohort. Am J Cardiol 2016;118:1888-1896.

13. Conte JV, Hermiller J Jr, Resar JR, et al. Complications after self-expanding transcatheter or surgical aortic valve replacement. Semin Thorac Cardiovasc Surg 2017;29:321-330.

14. Popma JJ, Adams DH, Reardon MJ, et al. Transcatheter aortic valve replacement using a self-expanding bioprosthesis in patients with 
severe aortic stenosis at extreme risk for surgery. J Am Coll Cardiol 2014;63:1972-1981.

15. Adams DH, Popma JJ, Reardon MJ, et al. Transcatheter aortic-valve replacement with a self-expanding prosthesis. N Engl J Med 2014; 370:1790-1798.

16. Leon MB, Piazza N, Nikolsky E, et al. Standardized endpoint definitions for transcatheter aortic valve implantation clinical trials: A consensus report from the valve academic research consortium. J Am Coll Cardiol 2011;57:253-269.

17. Beohar N, Doshi D, Thourani V, et al. Association of transcatheter aortic valve replacement with 30 -day renal function and 1-year outcomes among patients presenting with compromised baseline renal function: Experience from the PARTNER 1 trial and registry. JAMA Cardiol 2017;2:742-749.

18. Ferro $C J$, Chue $C D$, de Belder $M A$, et al. Impact of renal function on survival after transcatheter aortic valve implantation (TAVI): An analysis of the UKTAVI registry. Heart 2015;101:546-552.

19. lung B, Baron G, Butchart EG, et al. A prospective survey of patients with valvular heart disease in Europe: The euro heart survey on valvular heart disease. Eur Heart J 2003;24:1231-1243.

20. Oguri A, Yamamoto M, Mouillet G, et al. Impact of chronic kidney disease on the outcomes of transcatheter aortic valve implantation: Results from the France 2 registry. Eurolntervention 2015;10:e1-e9.

21. Williams M, Kodali SK, Hahn RT, et al. Sex-related differences in outcomes after transcatheter or surgical aortic valve replacement in patients with severe aortic stenosis: Insights from the PARTNER trial (placement of aortic transcatheter valve). J Am Coll Cardiol 2014;63: 1522-1528.

22. Lindman BR, Maniar HS, Jaber WA, et al. Effect of tricuspid regurgitation and the right heart on survival after transcatheter aortic valve replacement: Insights from the placement of aortic transcatheter valves II inoperable cohort. Circ Cardiovasc Interv 2015;8:e002073.

23. Cortes C, Amat-Santos IJ, Nombela-Franco L, et al. Mitral regurgitation after transcatheter aortic valve replacement: Prognosis, imaging predictors, and potential management. JACC Cardiovasc Interv 2016; 9:1603-1614.

24. Mehran R, Aymong ED, Nikolsky E, et al. A simple risk score for prediction of contrast-induced nephropathy after percutaneous coronary intervention: Development and initial validation. J Am Coll Cardiol 2004;44:1393-1399.

25. Sinning JM, Ghanem A, Steinhauser $\mathrm{H}$, et al. Renal function as predictor of mortality in patients after percutaneous transcatheter aortic valve implantation. JACC Cardiovasc Interv 2010;3:1141-1149.

26. McCullough PA, Wolyn R, Rocher LL, Levin RN, O'Neill WW. Acute renal failure after coronary intervention: Incidence, risk factors, and relationship to mortality. Am J Med 1997;103:368-375.

27. Toggweiler S, Gurvitch R, Leipsic J, et al. Percutaneous aortic valve replacement: Vascular outcomes with a fully percutaneous procedure. J Am Coll Cardiol 2012;59:113-118.

28. van Rosendael PJ, Delgado V, Bax JJ. Pacemaker implantation rate after transcatheter aortic valve implantation with early and new-generation devices: A systematic review. Eur Heart J 2018;39: 2003-2013.

\section{SUPPORTING INFORMATION}

Additional supporting information may be found online in the Supporting Information section at the end of the article.

How to cite this article: Pineda AM, Kevin Harrison J, Kleiman NS, et al. Clinical impact of baseline chronic kidney disease in patients undergoing transcatheter or surgical aortic valve replacement. Catheter Cardiovasc Interv. 2019;93: 740-748. https://doi.org/10.1002/ccd.27928 